See discussions, stats, and author profiles for this publication at: https://www.researchgate.net/publication/299974975

\title{
Exchange-bias-like effect of an uncompensated antiferromagnet
}

Article in Physical Review B · April 2016

DOI: 10.1103/PhysRevB.93.144406

4 authors, including:

\section{Bastian Henne \\ Infineon Technologies}

8 PUBLICATIONS 36 CITATIONS

SEE PROFILE

Mariano de Souza

São Paulo State University

69 PUBLICATIONS 720 CITATIONS

SEE PROFILE 


\title{
Exchange-bias-like effect of an uncompensated antiferromagnet
}

\author{
Bastian Henne, ${ }^{*}$ Verena Ney, Mariano de Souza, ${ }^{\dagger}$ and Andreas Ney \\ Institut für Halbleiter- und Festkörperphysik, Johannes Kepler Universität, Altenberger Str. 69, 4040 Linz, Austria
}

(Received 20 November 2015; revised manuscript received 18 March 2016; published 6 April 2016)

\begin{abstract}
The exchange bias effect is usually defined as horizontal shift of the field-cooled magnetization loop when an antiferromagnet is directly coupled to a ferromagnet. Uncompensated spins at the interface between the two layers are believed to cause this phenomenon. The presence of such, on the other hand, would infer a vertical, i.e., a magnetization-like shift stemming from the antiferromagnet. Observations of this effect are sparse, especially in the absence of a ferromagnet. We present a model system based on extremely Co doped $\mathrm{ZnO}$ in which the uncompensated spins of antiferromagnetic $\mathrm{Co}-\mathrm{O}-\mathrm{Co} \ldots$. . configurations lead to this vertical shift and therefore to a field-resistant magnetization. A simple Stoner-Wohlfarth-like model based on configurations of different sizes is used to explain the occurrence of this exchange-bias-like shift and a narrow opening of the magnetization curves.
\end{abstract}

DOI: 10.1103/PhysRevB.93.144406

\section{INTRODUCTION}

Antiferromagnetic spintronics envisions electronic devices which can be integrated at the nanoscale because they provide spin-dependent transport phenomena without having a finite magnetization $M$ [1-3]. The resulting insensitivity to magnetic fields $H$ makes their direct control difficult and alternative means as, e.g., coupling to a ferromagnet via exchange bias [4-6] are needed. Commonly, exchange biasing is evidenced by a field-like horizontal shift of the $M(H)$ loop dominated by the ferromagnet $[4,7,8]$. In contrast, its microscopic origin is attributed to uncompensated spins, i.e., an excess magnetization, of the antiferromagnet exchange coupled to the ferromagnet [6,9]. This infers the presence of an additional vertical shift [9]. Experimental observations of this shift are limited to few layered ferromagnet/antiferromagnet [10-16] or nanoparticulate/-composite systems [17-19]. Moreover, they are sparse in the absence of a ferromagnet and limited to few studies, e.g., on $\mathrm{NiO}$ [20] or maghemite nanoparticles [21]. Here we present a model system based on antiferromagnetic cobalt-doped $\mathrm{ZnO}$ (Co: $\mathrm{ZnO}$ ) where the uncompensated spins indeed exclusively lead to a vertical shift which is measurable by conventional magnetometry. Our findings pave the way for the exploration of the vertical exchange-bias-like effect in the absence of a ferromagnet and the possibility to achieve a finite field-resistent magnetization in an uncompensated antiferromagnet.

\section{MODEL SYSTEM}

Dilutely cobalt-doped $\mathrm{ZnO}$ was studied for decades [22,23] and more recently at higher concentrations in the prospect of obtaining room-temperature magnetic semiconductors [24]. In the wurtzite structure of $\mathrm{Co}: \mathrm{ZnO}$ each cation is tetrahedrally coordinated via oxygen to 12 nearest-cation-neighbors [see Fig. 1(a)], forming an hcp sublattice. It is well established that $\mathrm{Co}$ is substitutionally incorporated on $\mathrm{Zn}$ lattice sites

\footnotetext{
*bastian.henne@jku.at

†Permanent address: IGCE, Unesp-Univ Estadual Paulista, Departamento de Física, Cx. Postal 178, 13506-900, Rio Claro (SP), Brazil.
}

of the diamagnetic $\mathrm{ZnO}$ matrix [25] and that an isolated magnetic cation (single) acts as a paramagnetic impurity with a magnetic moment $\mu(\mathrm{Co})=3.4 \mu_{B}[22,26]$. Typically, neighboring magnetic atoms couple antiferromagnetically via oxygen by superexchange [24] and the next-cation-neighbor antiferromagnetic exchange coupling $J$ of Co-O-Co doubles in $\mathrm{Co}: \mathrm{ZnO}$ was determined to be $J / k_{B}=(15 \pm 3) \mathrm{K}$ [27]. For the limit of $100 \%$ Co in $\mathrm{ZnO}$, i.e., wurtzite $\mathrm{CoO}$, theory predicts that the system behaves as a fully compensated layered antiferromagnet with a Néel temperature $T_{N}$ close to $300 \mathrm{~K}$ [28]. While in most dilute magnetic semiconductors the solubility limit of the magnetic cation is in the low percentage range, doping levels up to at least $60 \%$ are feasible in $\mathrm{Co}: \mathrm{ZnO}$ without phase separation [29]. This opens the perspective to study more complex magnetic configurations at extreme doping levels providing a large number of uncompensated magnetic moments.

For large $\mathrm{Co}-\mathrm{O}-\mathrm{Co}-. .$. configurations in $\mathrm{Co}: \mathrm{ZnO}$ a plethora of different arrangements of the magnetic dopants are conceivable, some of which will be frustrated because the antiferromagnetic coupling cannot be satisfied for all atoms in tetrahedrally coordinated systems. For example, an open Co-O-Co-O-Co triple [Fig. 1(b)] has one uncompensated spin, i.e., it carries a reduced effective magnetic moment of $\frac{1}{3} \mu(\mathrm{Co})$, whereas frustrated spins occur in closed triples or tetrahedral quadruples which are indicated as blue arrows in Fig. 1. While a closed triple [Fig. 1(c)] also carries a reduced magnetic moment, the quadruple [Fig. 1(d)] is magnetically fully compensated. At higher dopant concentrations larger Co-O-Co-... configurations become increasingly important. Above the socalled coalescence limit of about $20 \%$ [30], connected Co paths can be found throughout the sample leading to long-range (antiferro)magnetic order [28]. Using Behringer's equations [31] one can calculate that for $60 \% \mathrm{Co:} \mathrm{ZnO}$ the abundance of singles, doubles, and triples together cover only $\sim 0.002 \%$ of all occurring configurations. An analytical description as in [27] of the magnetic behavior for large Co-O-Co-... configurations is therefore hardly possible. Despite the large number of magnetically compensated moments, a significant number of uncompensated spins at the edges of those large Co$\mathrm{O}-\mathrm{Co}-\ldots$ configurations will still be present. These remaining spins can respond to an external magnetic field during cooling 

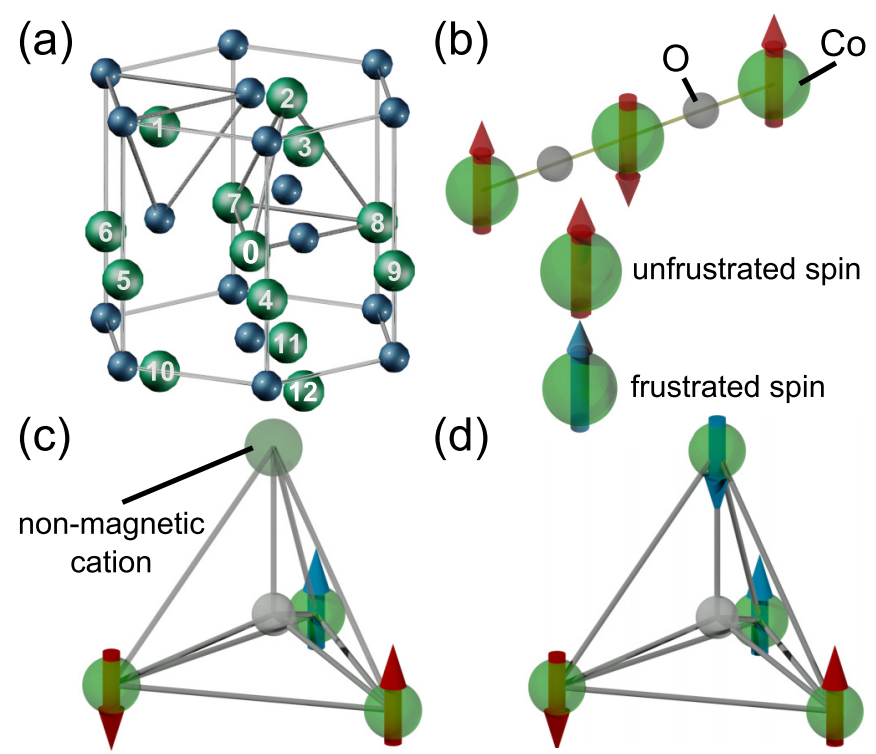

FIG. 1. Conceivable Co-O-Co-... configurations. (a) Wurtzite crystal structure with enumerated cations, (b) open triple with a net spin of $\frac{3}{2}$, (c) closed (and therefore frustrated) triple with spin $\frac{3}{2}$, and (d) frustrated Co tetrahedron with zero net moment. Frustrated spins are indicated in blue and unfrustrated in red.

across $T_{N}$ which results in a finite magnetization in an otherwise antiferromagnetically coupled system. This is in line with the fact that also antiferromagnetic nanoparticles behave ferrimagnetically because of uncompensated moments at their surface $[20,32]$. However, in the present case, uncompensated spins are not created by the finite size of the specimen but by finite magnetic dopant configurations in a thin film.

\section{EXPERIMENTAL DETAILS}

Epitaxial films of $\mathrm{Zn}_{0.4} \mathrm{Co}_{0.6} \mathrm{O}(60 \% \mathrm{Co}: \mathrm{ZnO})$ of high crystalline quality with a nominal thickness of $200 \mathrm{~nm}$ were grown by magnetron sputtering at $525^{\circ} \mathrm{C}$ on $\mathrm{Al}_{2} \mathrm{O}_{3}(001)$ substrates under ultrahigh-vacuum conditions $\left(p_{\text {base }} \sim 2 \times 10^{-9}\right.$ mbar, $p_{\text {process }} \sim 4 \times 10^{-3}$ mbar) using a stoichiometric $\mathrm{Co}_{3} \mathrm{O}_{4} / \mathrm{ZnO}$ composite target as described in more detail in [29]. X-ray absorption measurements to investigate the valency of the cations and to assure the phase pureness of the samples have already been carried out which also rule out the presence of metallic Co aggregations [29].

A commercial SQUID magnetometer (Quantum Design MPMS XL 5) was used to measure field and temperature dependent in-plane (IP) and out-of-plane (OOP) $M(H)$ curves and the following measurement sequence was applied: first the sample is cooled in $+5 \mathrm{~T}(\mathrm{pFC}$ ) from $300 \mathrm{~K}$ down to $2 \mathrm{~K}$ and two consecutive hysteresis cycles are recorded. Then the sample is heated up to $300 \mathrm{~K}$ and again field cooled to $2 \mathrm{~K}$, now at $-5 \mathrm{~T}$ (mFC). Here again two hysteresis cycles are measured. Afterwards, the sample is again heated up to $300 \mathrm{~K}$ and then cooled in nominally zero field (ZFC) down to $2 \mathrm{~K}$ to finally record the ZFC hysteresis loop. From all magnetization data the diamagnetic background of the substrate derived from high-field $M(H)$ data at $300 \mathrm{~K}$ was subtracted [25,27,33].

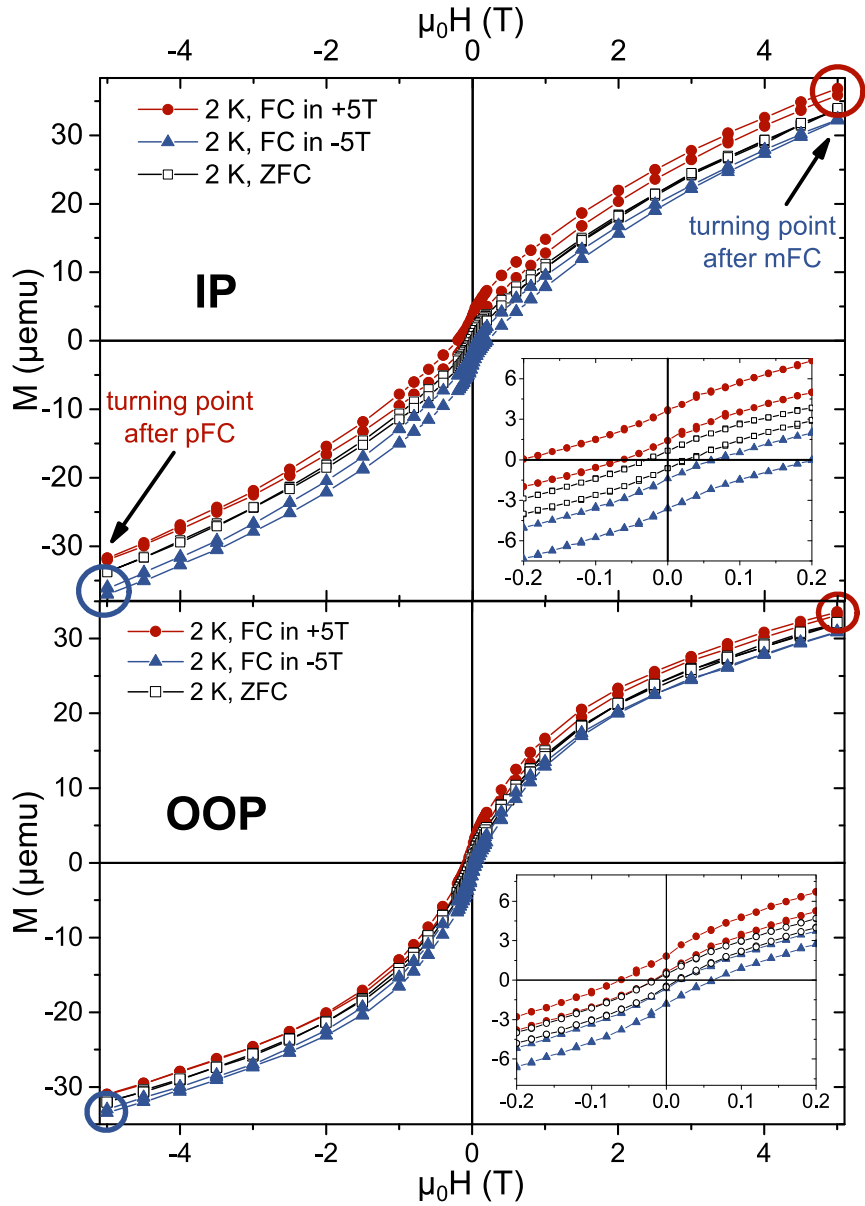

FIG. 2. IP (top) and OOP (bottom) $M(H)$ curves for plus, minus, and zero-field cooling ( $\mathrm{pFC}, \mathrm{mFC}$, and $\mathrm{ZFC}$, respectively). The low field region is enlarged in the insets. A cooling-field dependent vertical shift and an open hysteresis over a wide field range is observed (see text).

\section{RESULTS}

Figure 2 shows IP (top) and OOP (bottom) $M(H)$ curves for $60 \% \mathrm{Co:} \mathrm{ZnO}$ at $2 \mathrm{~K}$. Four key observations can be made.

(i) The $M(H)$ curves do not saturate up to $5 \mathrm{~T}$ and a strongly reduced effective magnetic moment of at most $0.1 \mu_{\mathrm{B}}$ per Co dopant can be estimated. Previous x-ray magnetic circular dichroism measurements have shown that even at $17 \mathrm{~T}$ no saturation is reached at $2 \mathrm{~K}$, corroborating antiferromagnetic coupling [29].

(ii) A clear but narrow hysteretic behavior extending over a wide field range is observed together with a finite remanence for the $\mathrm{pFC}, \mathrm{mFC}$, and even for the zero-field-cooled (ZFC) curves.

(iii) A vertical shift of the $\mathrm{pFC}$ and $\mathrm{mFC}$ curves with respect to the position of the ZFC hysteresis is visible which reverses with the direction of the cooling field being reminiscent of the exchange-bias effect.

(iv) A small gap is present in the $\mathrm{pFC}$ and $\mathrm{mFC} M(H)$ curves at the maximum field parallel to the cooling field direction (indicated by circles in Fig. 2) which vanishes after an additional magnetization cycle. 

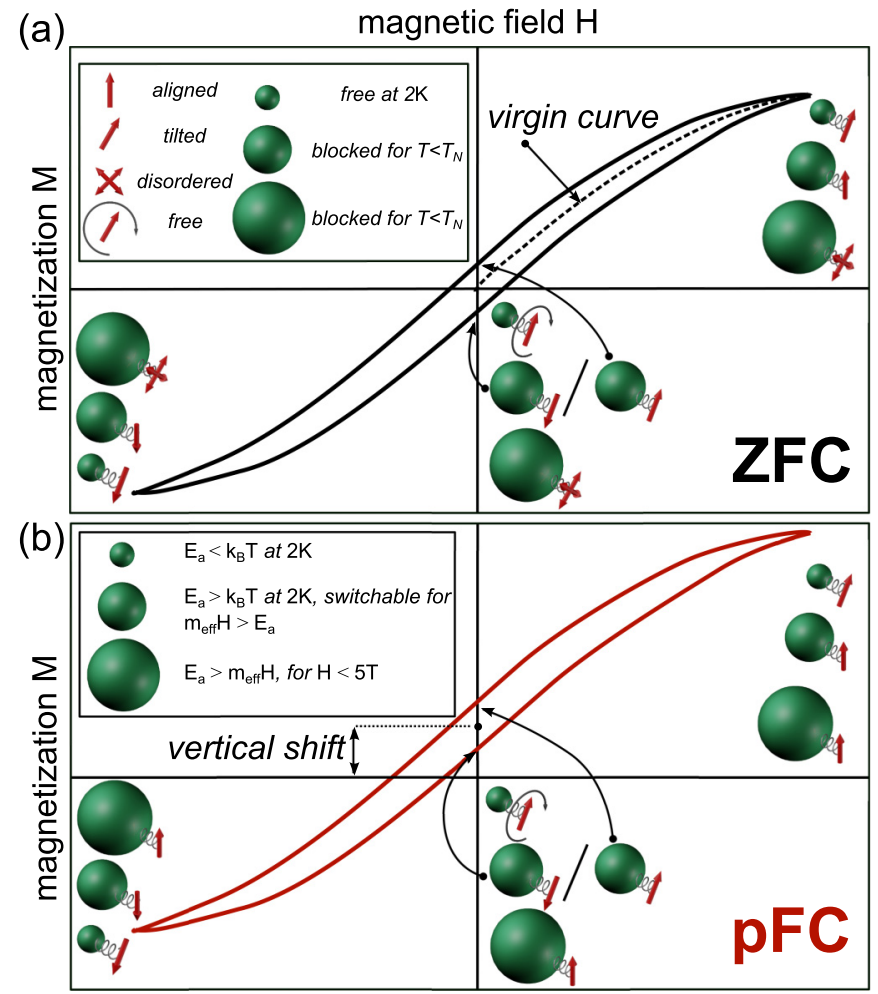

magnetic field $\mathrm{H}$

FIG. 3. Schematic model clarifying the occurrence of the hysteresis and the vertical shift categorizing three $\mathrm{Co}-\mathrm{O}-\mathrm{Co} \ldots$. . configuration sizes (green spheres). (a) In the ZFC case only small and mediumsized clusters contribute to the magnetic behavior and no shift occurs. (b) In the $\mathrm{pFC}$ case small and medium configurations contribute to the hysteresis and the large configurations cause the vertical magnetization shift ( $\mathrm{mFC}$ accordingly).

Observations (ii) and (iii) resemble previous observations on antiferromagnetic $\mathrm{NiO}$ nanoparticles [20] which are not expected for a fully compensated antiferromagnet. They can be understood by the schematic model shown in Fig. 3 .

Uncompensated magnetic moments $m_{\text {eff }}$ (red arrows) are exchange coupled to the remaining, magnetically fully compensated Co-O-Co-... configuration (green spheres). The latter are divided into three size categories [34] using a Stoner-Wohlfarth-like model [35]: the gain in Zeeman energy $E_{\mathrm{Z}} \sim m_{\mathrm{eff}} H$ can be compared to an anisotropy energy $E_{\mathrm{a}} \sim$ $K \cdot V$ with an effective anisotropy constant $K$ and volume $V$ leading to a switching barrier for $m_{\text {eff }}$. Note that in contrast to the classical Stoner-Wohlfarth model, here only $E_{Z}$ is associated with $m_{\text {eff }}$, while $E_{\mathrm{a}}$ is associated with the remaining antiferromagnetically compensated part of the Co-O-Co- ... configuration and not with $m_{\text {eff }}$ itself. For small configurations $K \cdot V$ is smaller than the thermal energy $k_{\mathrm{B}} T$ at $2 \mathrm{~K}$. The magnetic moment $m_{\text {eff }}$ therefore can freely fluctuate as in superparamagnetic particles and aligns with $H$ leading to an anhysteretic, paramagnetic-like contribution. For the mediumsized configurations, however, $E_{\mathrm{a}}$ is larger than $k_{\mathrm{B}} T$ at $2 \mathrm{~K}$. Therefore, those configurations are blocked in their respective magnetization state. Their magnetization can be switched with $H<5 \mathrm{~T}$ once $m_{\text {eff }} H$ becomes larger than $K \cdot V$. Large configurations, in contrast, are not switchable by $H$ up to $5 \mathrm{~T}$, because $E_{\mathrm{Z}}$ is always smaller than $E_{\mathrm{a}}$. Coupling between the individual clusters is neglected in this simple model.

The presence of a hysteresis in the $M(H)$ curves, even under ZFC, [observation (ii)] can be understood as follows: when the sample is cooled down in zero field across $T_{N}$, the net moments $m_{\text {eff }}$ of the medium and large configurations are blocked in random directions due to thermal disorder, while the small ones remain freely fluctuating at $2 \mathrm{~K}$. Note, however, that the specimen has no sharp $T_{N}$ since each configuration possesses its own $T_{N}$ depending on size and local environment ranging from $15 \mathrm{~K}$ (pairs) up to just below $300 \mathrm{~K}$ (bulk) [28]. When $H$ is now increased to $+5 \mathrm{~T}$ (virgin curve in Fig. $3 \mathrm{ZFC}$ ), all $m_{\mathrm{eff}}$ belonging to the small configurations gradually align parallel with $H$, whereas medium-sized configurations successively switch depending on their respective volume. When $H$ is decreased again, the small configurations return to their thermally fluctuating state while the medium-sized configurations partially retain their aligned state because $E_{\text {a }}$ prevents them from switching back in their initially disordered state. This results in a small magnetic remanence and hysteresis. Large configurations remain in their initial disordered state throughout the entire $M(H)$ loop.

The shift of the $\mathrm{p} / \mathrm{mFC} M(H)$ curves [observation (iii)], on the other hand, can be understood by taking into account the magnetic behavior of the large configurations. Because $E_{\mathrm{a}}>E_{\mathrm{Z}}, m_{\text {eff }}$ can only be aligned parallel to the external field when $E_{a}$ becomes sufficiently small, which happens close to $T_{N}$ at which $K=0$. Thus large configurations cooled through $T_{N}$ in a magnetic field gain a finite imprinted magnetization which adds to the samples' ZFC magnetization. Well below $T_{N}$ this imprinted magnetization leads to a vertical shift of the entire hysteresis, which is nonvolatile. This shift is describable as vertical exchange-bias-like effect being present in the absence of a ferromagnet; an observation quite distinct from those made in common exchange-bias systems. Even in bulk compensated ferrimagnetic hosts with ferrimagnetic clusters a pure horizontal shift was recently observed [36].

As pointed out by Geshev [37] it is crucial to check that no minor hysteresis loop is misinterpreted as a vertical exchange-bias effect. In our measurements a small gap at the maximum magnetic field in the cooling field direction [observation (iv)] demonstrates that the system is initially not in the reversible regime. Therefore, two consecutive hysteresis loops were measured at $2 \mathrm{~K}$ for $\mathrm{pFC}$ and $\mathrm{mFC}$.

Figure 4 displays the pFC $M(H)$ curves in the IP (top) and OOP (bottom) direction at $2 \mathrm{~K}$. The high-field region at $5 \mathrm{~T}$ which is parallel to the cooling field is enlarged in the insets. One can see that the initial gap of the $M(H)$ curves seen also in Fig. 2 has disappeared after the second hysteresis cycle. This assures that the system is now in the reversible regime for $|H|<5 \mathrm{~T}$. To visualize the irreversible contribution, the direct difference of the two consecutive $M(H)$ curves is taken and shown in Fig. 4 as black open squares. The difference between the first and second $M(H)$ loop amounts to roughly $1.1 \mu \mathrm{emu}$ for IP, while in the OOP direction the difference of $0.7 \mu \mathrm{emu}$ is significantly smaller. Initially, the difference remains constant as long as $H$ is parallel to the cooling-field direction. Obviously, the reduction of $E_{Z}$ does not lead to a reduction of $m_{\mathrm{eff}}$. As soon as the applied magnetic field changes its sign the difference 


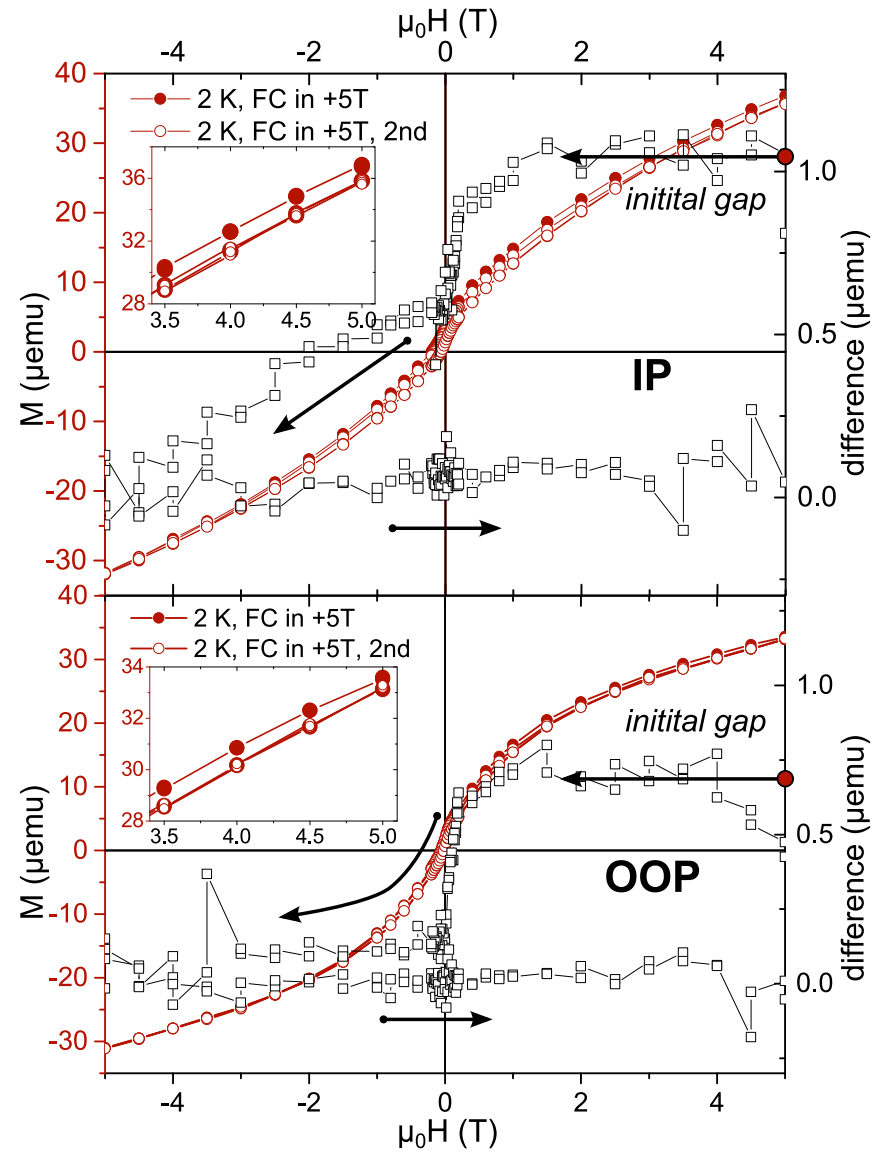

FIG. 4. Two consecutive $M(H)$ curves after $\mathrm{pFC}$ for the IP (top) and OOP (bottom) direction. The respective high-field region is enlarged in the insets. The difference between the two measurements is plotted as black open squares (right scale). The size of the initial gap is marked and the direction of change is indicated by arrows.

slowly decreases to zero around $-4 \mathrm{~T}$ in the IP direction. In contrast, for the OOP direction this relaxation happens much more abruptly at much smaller negative fields. In the second half of the $M(H)$ cycle the difference remains zero, i.e., no further relaxation occurs. To picture this behavior one has to consider that the uncompensated moments $m_{\text {eff }}$ are composed of partially frustrated, i.e., metastable, complex arrangements of a number of individual Co spins, some of which may even be in a noncollinear state. Those complex arrangements, however, attain a preferential direction imprinted during FC through $T_{N}$. When reversing the field during the $M(H)$ cycle these spins rearrange to satisfy the altered $E_{Z}$ leading to a net reduction of $m_{\text {eff }}$. The measured magnetization, which corresponds to the sum over all $m_{\text {eff }}$, therefore becomes smaller after completing the $M(H)$ loop. The second hysteresis starts in this reduced-moment state and therefore returns to the very same point after the second $M(H)$ cycle. It is noteworthy that although the influence of field-imprinted frustration is minimized for the second $M(H)$ loop, the overall vertical shift remains unaffected. This suggests that field-imprinted frustration is relevant for the similar behavior known as training effect in ordinary exchange-bias systems [6].

Finally, to quantify the size of the vertical shift, the second $\mathrm{p} / \mathrm{mFC}$ IP and OOP hystereses at $2 \mathrm{~K}$ are vertically translated

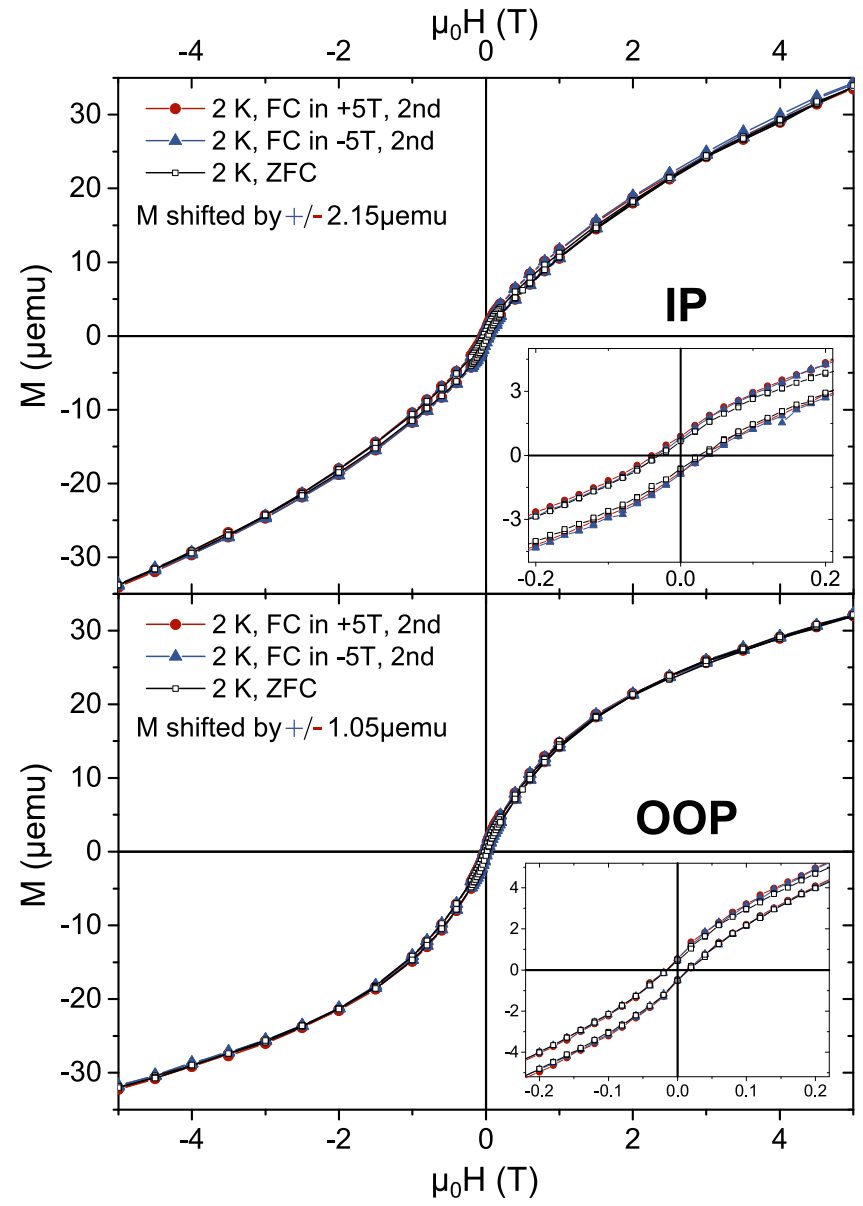

FIG. 5. Vertically shifted FC $M(H)$ curves for the IP (top) and OOP (bottom) direction. In the insets the region at low magnetic field is enlarged.

to match the ZFC curve as shown in Fig. 5. This leads to an almost perfect replication of the ZFC curve and it was checked that a horizontal shift cannot reproduce the ZFC curves comparably well (not shown). This supports the above interpretation of observation (iii) that the (antiferromagnetically) stabilized uncompensated spins $m_{\text {eff }}$ of the large Co-O-Co-... configurations lead to an offset magnetization parallel to the cooling field rather than a horizontal shift of the hysteresis along the field axis. This vertical shift corresponds to the sum over all field-imprinted $m_{\text {eff }}$ of the large configurations and is significantly different for IP $(2.15 \mu \mathrm{emu})$ and OOP $(1.05 \mu \mathrm{emu})$. This anisotropy, not to be mistaken with $E_{\mathrm{a}}$, is similar to the anisotropic "demagnetization" in Fig. 4. Obviously, the IP direction is the easy-plane and OOP the hard-axis direction. Anisotropic paramagnetism for dilute samples is well established. A single ion anisotropy $D\left(D / k_{\mathrm{B}} \approx 4 \mathrm{~K}\right)$ stemming from the anisotropic crystal field with an easy $a$ plane and hard $c$ axis (positive sign of $D$ ) is reported in the literature [22,23,26,33]. Obviously, the sign of the single-spin anisotropy $D$ is maintained for the uncompensated antiferromagnet identifying both $\mathrm{Co}: \mathrm{ZnO}$ as easy $a$-plane systems. The anisotropy between IP and OOP can be pictured by assuming a different size of $m_{\text {eff }}$ for the medium and large configurations, respectively. While during FC in 
the easy-plane the effective anisotropy field helps realizing arrangments with large $m_{\text {eff }}$, it opposes its formation during FC along the hard-axis direction.

\section{CONCLUSION}

A model system based on uncompensated spins in antiferromagnetically coupled $\mathrm{Co}-\mathrm{O}-\mathrm{Co}-\ldots$ configurations in a $\mathrm{ZnO}$ crystal matrix has been presented to study the properties of uncompensated antiferromagnetism, especially with regard to the microscopic origin of the exchange-bias effect in the absence of coupling to a ferromagnet. A narrow hysteresis over a wide field range leading to a finite remanence was observed in all $M(H)$ curves. In addition, a cooling-field induced vertical shift occurs. Furthermore, the contribution of frustration could be quantified by measuring two consecutive hystereses loops after FC. Both effects exhibit an easy $a$-plane anisotropy in accordance with the single spin anisotropy of the dilute system. A concentration dependence of the observed effects was found and will be discussed together with the temperature dependence (the effect vanishes around $15 \mathrm{~K}$ ) elsewhere. A similar effect has been observed in an ensemble of antiferromagnetic $\mathrm{NiO}$ nanoparticles with a broad size distribution [20]. We expect it to be observable in other highly uncompensated antiferromagnetic materials, like in layers of ternary oxides (perovskites or spinels) with finite site disorder and anticipate possible applications in the field of antiferromagnetic spintronics.

\section{ACKNOWLEDGMENTS}

The authors gratefully acknowledge funding by the Austrian Science Fund (FWF), Project No. P26164-N20. The authors thank R. Koch and S. Müllegger for helpful discussions.
[1] X. Marti, D. Yi, J. Liu, J. H. Chu, C. Rayan-Serrao, S. Suresha, A. B. Shick, J. Zelezný, T. Jungwirth, J. Fontcuberta, and R. Ramesh, Nat. Commun. 5, 4671 (2014).

[2] E. V. Gomonay and V. M. Loktev, Low Temp. Phys. 40, 17 (2014).

[3] X. Marti, F. Ignasi, and T. Jungwirth, IEEE Trans. Magn. 51, 2900104 (2015)

[4] W. P. Meiklejohn and C. P. Bean, Phys. Rev. 102, 1413 (1956).

[5] J. Nogués and I. K. Schuller, J. Magn. Magn. Mater. 192, 203 (1999).

[6] M. Kiwi, J. Magn. Magn. Mater. 234, 584 (2001).

[7] M. Gibert, P. Zubko, R. Scherwitzl, J. Íñiguez, and J.-M. Triscone, Nat. Mater. 11, 195 (2012).

[8] E. Lage, C. Kirchhof, V. Hrkac, L. Kienle, R. Jahns, R. Knöchel, E. Quandt, and D. Meyners, Nat. Mater. 11, 523 (2012).

[9] H. Ohldag, A. Scholl, F. Nolting, E. Arenholz, S. Maat, A. T. Young, M. Carey, and J. Stöhr, Phys. Rev. Lett. 91, 017203 (2003).

[10] J. Nogués, C. Leighton, and I. K. Schuller, Phys. Rev. B 61, 1315 (2000).

[11] H. Ohldag, A. Scholl, F. Nolting, S. Anders, F. U. Hillebrecht, and J. Stöhr, Phys. Rev. Lett. 86, 2878 (2001).

[12] Z. Y. Liu, Appl. Phys. Lett. 85, 4971 (2004).

[13] H. Ohldag, H. Shi, E. Arenholz, J. Stöhr, and D. Lederman, Phys. Rev. Lett. 96, 027203 (2006).

[14] M. Gruyters and D. Schmitz, Phys. Rev. Lett. 100, 077205 (2008).

[15] Y. Fan, K. J. Smith, G. Lüpke, A. T. Hanbicki, R. Goswami, C. H. Li, H. B. Zhao, and B. T. Jonker, Nat. Nanotechnol. 8, 438 (2013).

[16] R. Rana, P. Pandey, R. P. Singh, and D. S. Rana, Sci. Rep. 4, 4138 (2014).

[17] A. N. Dobrynin, D. N. Ievlev, K. Temst, P. Lievens, J. Margueritat, J. Gonzalo, C. N. Afonso, S. Q. Zhou, A. Vantomme, E. Piscopiello, and G. Van Tendeloo, Appl. Phys. Lett. 87, 012501 (2005).

[18] Z. M. Tian, S. L. Yuan, S. Y. Yin, L. Liu, J. H. He, H. N. Duan, P. Li, and C. H. Wang, Appl. Phys. Lett. 93, 222505 (2008).
[19] X. Sun, N. F. Huls, A. Sigdel, and S. Sun, Nano Lett. 12, 246 (2012).

[20] R. H. Kodama, S. A. Makhlouf, and A. E. Berkowitz, Phys. Rev. Lett. 79, 1393 (1997).

[21] H. Khurshid, W. Li, M.-H. Phan, P. Mukherjee, G. C. Hadjipanayis, and H. Srikanth, Appl. Phys. Lett. 101, 022403 (2012).

[22] T. L. Estle and M. de Wit, Bull. Am. Phys. Soc. 6, 445 (1961).

[23] P. Koidl, Phys. Rev. B 15, 2493 (1977).

[24] J. M. D. Coey, in Handbook of Spin Transport and Magnetism, edited by E. Y. Tsymbal and I. Zutic (CRC Press, Boca Raton, FL, 2011).

[25] A. Ney, M. Opel, T. C. Kaspar, V. Ney, S. Ye, K. Ollefs, T. Kammermeier, S. Bauer, K.-W. Nielsen, and S. T. B. Goennenwein, New J. Phys. 12, 013020 (2010).

[26] P. Sati, R. Hayn, R. Kuzian, S. Régnier, S. Schäfer, A. Stepanov, C. Morhain, C. Deparis, M. Laügt, M. Goiran, and Z. Golacki, Phys. Rev. Lett. 96, 017203 (2006).

[27] A. Ney, V. Ney, F. Wilhelm, A. Rogalev, and K. Usadel, Phys. Rev. B 85, 245202 (2012).

[28] S. K. Nayak, M. Ogura, A. Hucht, H. Akai, and P. Entel, J. Phys.: Condens. Matter 21, 064238 (2009).

[29] B. Henne, V. Ney, K. Ollefs, F. Wilhelm, A. Rogalev, and A. Ney, Sci. Rep. 5, 16863 (2015).

[30] C. Lorenz, R. May, and R. M. Ziff, J. Stat. Phys. 98, 961 (2000).

[31] R. E. Behringer, J. Chem. Phys. 29, 537 (1958).

[32] L. Néel, Proc. Phys. Soc. A 65, 869 (1952).

[33] A. Ney, T. Kammermeier, K. Ollefs, S. Ye, V. Ney, T. C. Kaspar, S. A. Chambers, F. Wilhelm, and A. Rogalev, Phys. Rev. B 81, 054420 (2010).

[34] Note that "size" refers to the number of directly connected, nearest-cation-neighbor Co dopant atoms which in principle can exist in any shape from a chain to an almost spherical arrangement.

[35] E. C. Stoner and E. P. Wohlfarth, Philos. Trans. R. Soc. A 240, 599 (1948)

[36] A. K. Nayak, M. Nicklas, S. Chadov, P. Khuntia, C. Shekhar, A. Kalache, M. Baenitz, Y. Skourski, V. K. Guduru, A. Puri, U. Zeitler, J. M. D. Coey, and C. Felser, Nat. Mater. 14, 679 (2015).

[37] J. Geshev, J. Magn. Magn. Mater. 320, 600 (2008). 